\title{
Misplaced IUCD in bladder - A case report
}

\author{
Anupa Singhal ${ }^{1 *}$, Puneeta Mahajan ${ }^{2}$, Prachi Renjhen ${ }^{3}$, Dolly Chawla ${ }^{4}$, Neeru Malik ${ }^{5}$ \\ ${ }^{1}$ Senior Medical Officer, ${ }^{2}$ Senior Specialist, ${ }^{3}$ Professor, ${ }^{4}$ Senior Specialist and HOD, ${ }^{5}$ Chief Medical Officer, Department of Obstetrics and \\ Gynaecology, ${ }^{1-5}$ Dr. Baba Saheb Ambedkar Medical College and Hospital, Delhi, India
}

Corresponding Author: Anupa Singhal

Email: anupasinghal@gmail.com

Received: $13^{\text {th }}$ January, 2019

Accepted: $4^{\text {th }}$ March, 2019

\begin{abstract}
Intrauterine contraceptive device (IUCD) is the most widely used reversible method of contraception with minimal side effect. Uterine perforation is an uncommon complication of IUCD insertion and occurs in 1 in1000 insertion. Because of close proximity of urinary bladder to the uterus, an IUCD in rare incidence can perforate or migrate into urinary bladder. We report a case of misplaced IUCD in bladder. A combination of pelvic X-ray, 2D and 3D sonography was used to identify a missing IUCD. IUCD was successfully removed cystoscopically without any complication.
\end{abstract}

Keywords: IUCD, Misplaced, Bladder.

\section{Introduction}

Intrauterine contraceptive device (IUCD) is the most widely used reversible method of contraception all over the world. In India it is available free of cost in government hospitals. The device insertion is practiced at various health care centers throughout India by the paramedical staff. Although a safe procedure of insertion is practiced, a rare chance of perforation exists during insertion especially by personnel of variable skill. We report a case of misplaced IUCD in bladder.

\section{Case Report}

A 36 years old woman $\mathrm{P}_{2} \mathrm{~L}_{2} \mathrm{~A}_{2}$ presented to our outpatient department with dysuria and pain in lower abdomen for fifteen days. Her ultrasound report showing IUCD in the urinary bladder. She had a history of insertion of IUCD four years back by a midwife when her youngest child was one year old. After insertion of IUCD there was persistent pain in abdomen for two weeks for which she took analgesics. After two months when she was examined by midwife it was found that IUCD thread was missing and her urine for pregnancy test was positive. Ultrasonography showed intrauterine pregnancy of five weeks and no IUCD in uterine cavity. Pelvic x-ray was not done. It was assumed that IUCD had been expelled spontaneously though she did not give any history of expulsion. Medical Termination of pregnancy was done. She again got pregnant after two years and underwent medical termination of pregnancy. This time no ultrasound was done. She used combined oral pills as contraceptive thereafter. For last fifteen days she developed dysuria and pain in abdomen for which pelvic ultrasound done, which detected IUCD in bladder which was missed in previous scan (Fig. 1). Physical and pelvic examination did not reveal any significant findings except mild tenderness at the suprapubic region. Urinalysis showed 2-3 pus cells. Urine culture was sterile. 3-D ultrasound revealed one limb of IUCD embedded in bladder wall, which finding confirmed on plain CT pelvis. After localizing the IUCD on imaging, cystoscopy was done, which showed one centimetre of IUCD in the posterior bladder wall away from ureteric orifice (Fig. 2). IUCD was removed by endoscopic forceps. No perforation appreciated in bladder wall. As the woman wanted permanent method of contraception, laparoscopic sterilization was done. In order to facilitate complete healing, an indwelling, transurethral Foley's catheter was placed for twenty-one days. Post-operative period was uneventful. On follow-up, there were no urinary complaints.

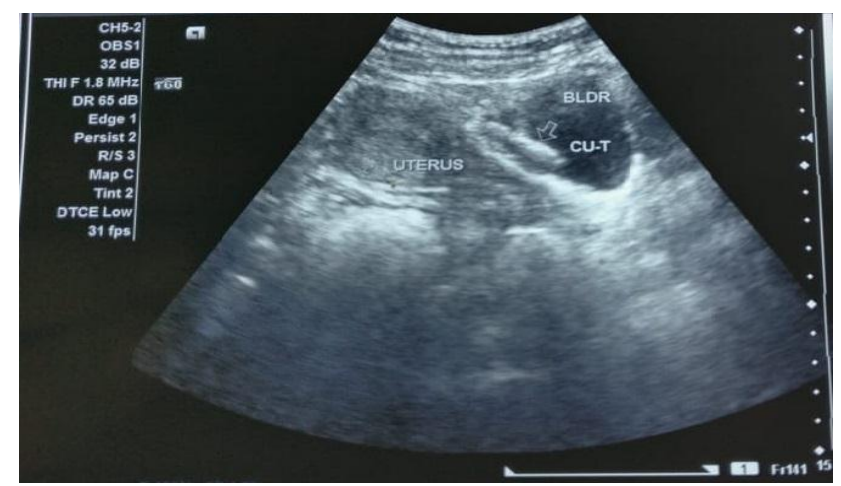

Fig. 1: Sonographic image showing IUCD inside bladder

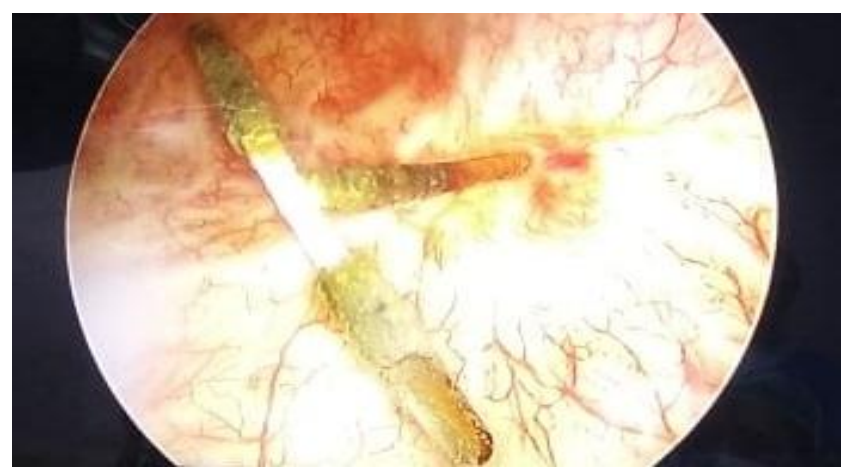

Fig. 2: Photograph showing cystoscopic view of IUCD inside bladder 


\section{Discussion}

IUCD is an effective, safe and economic method of contraception and is used by $13.9 \%$ of the world's 1.16 billion women aged 15-49 years. ${ }^{1}$ Uterine perforation is an uncommon complication of intrauterine device insertion, with an incidence of 1 in 1000 insertion. Very rarely, a device may perforate into bowel or urinary tract. Because of close proximity of urinary bladder to the uterus, an IUCD in rare incidence can perforate or migrate into urinary bladder.

There are more than 70 cases of perforation involving the urinary tract reported in literature. ${ }^{1}$ Most of the patients in these cases experienced urinary symptoms, and half of the reported cases resulted in stone formation around the IUCD. ${ }^{2}$ Bladder perforation should be considered whenever a patient with a "missing" IUCD presents with urinary symptoms. In our case intravesical IUCD was diagnosed only after the urinary complaints.

Risk factors for uterine perforation include clinician inexperience in IUCD placement, or immobile uterus, a retroverted uterus, and the presence of a myometrial defect (pre-existing or created during the procedure by uterine sound or IUCD inserter). Most of perforation occurs at the time of insertion. ${ }^{3}$ Uterine perforation can often be asymptomatic and are not identified until months or years after insertion. Symptoms suggesting perforation include pain, cramping, irregular bleeding, dyspareunia, and absent string. ${ }^{2}$ In the present case uterine perforation could have occurred at the time of insertion as IUCD inserted by inexperienced midwife or due to extreme posterior position of uterus.

Another important complication of IUCD insertion is expulsion and occurs in 3 to 10 percent of women. If the strings of IUCD are not visible, complete expulsion may have occurred. The diagnosis of complete expulsion requires ultrasound confirmation that the IUCD is not in the uterus, followed by x-ray documentation that IUCD is not in the abdomen or pelvis. Expulsion cannot be diagnosed without $\mathrm{x}$-ray documentation, unless the expulsion was noted by the user. It is usually impossible to detect an IUCD that is located outside the uterus with ultrasound. ${ }^{3}$
Ultrasound scanning is a better modality for identifying devices that are intrauterine rather than extrauterine. ${ }^{1}$ In our case missing thread on per-vaginal examination was not investigated and assumed as expulsion though patient did not give any history of expulsion.

IUCD which has perforated or migrated into the urinary bladder must be removed even if it is asymptomatic. This is to prevent complications such as calculus formation and bladder rupture. An IUCD which has migrated into urinary bladder is treated by cystoscopic removal or by open suprapubic cystotomy. Cystoscopic removal is preferred because it has low morbidity and is highly effective. ${ }^{4}$ In this case cystoscopic removal was done successfully.

\section{Conclusion}

Regular training of doctors and paramedics can prevent complications associated with insertion of IUCD. Postinsertion follow-up visits are important and missing threads should be evaluated meticulously. Urinary complaints with not retrieved IUCD should raise suspicion of intravesical IUCD.

\section{Conflict of Interest: None.}

\section{References}

1. Rowlands S, Oloto E, Horwell DH. Intrauterine devices and risk of uterine perforation: current perspectives. Open Access $J$ Contracept 2016;7:19-32.

2. Nilsestuen L. IUD Perforation to the Urinary Bladder: Ultrasonographic Diagnosis. J Diagn Med Sonography 2013;29(3):126-129. Doi: 10.1177/8756479313477732

3. Dean G, Goldberg AB. Management of problems related to intrauterine contraception. https://somepomed.org/articulos/contents/mobipreview.htm?22 /34/23073

4. Gyasi-Sarpong CK, ManuMaison PO, Morhe E. Intravesical migration of an intrauterine device. BMC Res Notes 2016;9:4.

How to cite this article: Singhal A, Mahajan P, Renjhen P, Chawla D, Malik N, Misplaced IUCD in bladder - A case report. Indian J Obstet Gynecol Res 2019;6(2):220-221. 